\title{
A NOTE ON A CLASS OF SUBMULTIPLICATIVE FUNCTIONS
}

\author{
P. SURYA MOHAN \\ Department of Mathematics, Indian Institute of Technology, Kharagpur, West Bengal 721302, India \\ e-mail: surya_tc@yahoo.co.in
}

(Received 14 January, 2003; accepted 2 September 2003)

\begin{abstract}
In 1989, Alladi, Erdös and Vaaler confirmed their own conjecture about a class of multiplicative functions by means of a deep result of Baranyai on hypergraphs. In this note we give a simple direct proof of the result which is derived in their proof as a consequence of the above mentioned graph theoretic result.
\end{abstract}

2000 Mathematics Subject Classification. 11N64.

1. Introduction. In 1984, Alladi, Erdös and Vaaler considered the following conjecture.

CONJECTURE. Let $n$ be a square-free integer and $h$ a multiplicative function satisfying $0 \leq h(p) \leq 1 /(k-1)$ on primes $p$, where $k$ is a natural number. Then

$$
\sum_{d \mid n} h(d) \leq c_{k} \sum_{d \mid n, d \leq n^{1 / k}} h(d),
$$

where $c_{k}$ denotes a constant depending only on $k$.

Later in [1], they proved the above conjecture, using a result (namely, the following Proposition) which is a special case of a theorem of Baranyai on hypergraphs [2]. Thus, in view of [3], the above statement automatically holds even when $h$ is a submultiplicative function. In the sequel, we use $p$ (with or without suffixes) to denote primes.

Proposition. Let $k(\geq 1)$ and $\ell(\geq 0)$ be given integers. Suppose $N=p_{1} p_{2} p_{3} \ldots p_{k \ell}$, with $p_{1}<p_{2}<p_{3}<\ldots<p_{k \ell}$. Then the number of $d$, such that $d \mid N, d \leq N^{\frac{1}{k}}$ and having exactly $\ell$ prime divisors, is at least

$$
\frac{1}{k}\left(\begin{array}{c}
k \ell \\
\ell
\end{array}\right) \text {. }
$$

2. Proof of the Proposition. For $\ell=0$, the Proposition holds trivially. Let $S_{k \ell}=\{1,2,3, \ldots, k \ell\}$. For any permutation $\pi=\left\{\sigma_{1}, \sigma_{2}, \sigma_{3}, \ldots \sigma_{k \ell}\right\}$ of $S_{k \ell}$, set $\xi_{\pi}=$ $\left\{A_{1}, A_{2}, A_{3}, \ldots A_{k}\right\}$ where $A_{j}=\left\{\sigma_{(j-1) \ell+1}, \ldots \sigma_{j \ell}\right\}$. For every B with $|B|=\ell$, let $\delta_{\pi}(B)$ denote 1 if $B \in \xi_{\pi}$, or 0 otherwise. For each subset $A$ of $S_{k \ell}$, there is an associated divisor $d_{A}$ of $N$ given as the product of all primes $p_{i}$, with $i \in A$, and this association is a bijection. Let $\zeta_{1}$ be the collection of all subsets $A$ of $S_{k \ell}$, such that $|A|=\ell$ and $d_{A} \leq N^{\frac{1}{k}}$. Similarly let $\zeta_{2}$ be the collection of all subsets $B$ of $S_{k \ell}$, such that $|B|=\ell$ and $d_{B}>N^{\frac{1}{k}}$. Since any $C \subseteq S_{k \ell}$ having $\ell$ elements belongs to exactly one of $\zeta_{1}$ or $\zeta_{2}$, 
we have

$$
\left|\zeta_{1}\right|+\left|\zeta_{2}\right|=\left(\begin{array}{c}
k \ell \\
\ell
\end{array}\right)
$$

Since $\prod_{j} d_{A_{j}}=N, \exists A_{j}$ such that $d_{A_{j}} \leq N^{\frac{1}{k}}$, and so we have for every permutation $\pi$,

$$
\left|\zeta_{2} \cap \xi_{\pi}\right| \leq(k-1)\left|\zeta_{1} \cap \xi_{\pi}\right|,
$$

which can be written as

$$
\sum_{B \in \zeta_{2}} \delta_{\pi}(B) \leq(k-1) \sum_{A \in \zeta_{1}} \delta_{\pi}(A) .
$$

Now summing over all $\pi$, we get

$$
\sum_{B \in \zeta_{2}} \sum_{\pi} \delta_{\pi}(B) \leq(k-1) \sum_{A \in \zeta_{1}} \sum_{\pi} \delta_{\pi}(A) .
$$

Since for any $C$ with exactly $\ell$ elements, $\sum_{\pi} \delta_{\pi}(C)$ being $k$ times $\ell$ ! $(k \ell-\ell)$ ! is independent of $\mathrm{C}$, the above inequality leads to

$$
\left|\zeta_{2}\right| \leq(k-1)\left|\zeta_{1}\right|
$$

From (1) and (2), we obtain

$$
k\left|\zeta_{1}\right| \geq\left(\begin{array}{c}
k \ell \\
\ell
\end{array}\right)
$$

which completes the proof of the proposition.

ACKNOWLEDGEMENTS. This work was done at TIFR centre at Bangalore, during the summer visit 2002-2003. I am very grateful to TIFR for the facilities that were provided.

\section{REFERENCES}

1. K. Alladi, P. Erdös and J. D. Vaaler, Multiplicative functions and small divisors, II, J. Number Theory 31 (1989), 183-190.

2. Z. S. Baranyai, On the factorization of the complete uniform hyper graph, in Colloquia Mathematica Societatis Janos Bolyai, 10 (Keszthely, Hungary, 1973), 91-102.

3. S. Srinivasan, On an arithmetical inequality, Glasgow Math. J. 36 (1994), 81-86. 